\title{
Effect of prosthetic design on center of pressure excursion in partial foot prostheses
}

Michael P. Dillon, PhD, BPO(Hons); ${ }^{*}$ Stefania Fatone, PhD, BPO(Hons); ${ }^{2}$ Andrew H. Hansen, PhD $^{2-3}$

${ }^{1}$ National Centre for Prosthetics and Orthotics, Musculoskeletal Research Centre, La Trobe University, Bundoora, Victoria, Australia; ${ }^{2}$ Northwestern University Prosthetics-Orthotics Center, and Department of Physical Medicine and Rehabilitation, Feinberg School of Medicine, Northwestern University, Chicago, IL; ${ }^{3}$ Minneapolis Department of Veterans Affairs Health Care System, Minneapolis, MN

\begin{abstract}
Investigations into the gait of persons with partial foot amputation (PFA) suggest that the effective foot length can be restored when the prosthesis incorporates a relatively stiff forefoot, restricts dorsiflexion, and includes a mechanism whereby forces caused by loading the toe lever can be comfortably distributed to the leg (e.g., an anterior tibial shell). The purpose of this investigation was to systematically alter these variables to understand which design elements are responsible for restoration of the effective foot length. By manipulating features of the prosthesis design in two persons with PFA, we demonstrated using three-dimensional motion analysis that the prosthesis must incorporate each of these design elements to restore the effective foot length. When these design elements were used in concert, the persons with PFA adopted a gait pattern more consistent with nondisabled persons. Further work is required on a larger cohort to ensure the observations are generalizable.
\end{abstract}

Key words: amputation, artificial limb, center of pressure, foot length, gait, Lisfranc, partial foot, prosthesis, prosthetic design, rehabilitation, transmetatarsal.

\section{INTRODUCTION}

For much of the last 4 decades, our understanding of the gait of persons with partial foot amputation (PFA) and the influence of prosthetic intervention was based on theoretical force analyses [1-4]. These analyses stemmed from an appreciation of the gait of persons without limb loss and led to the assumption that partial foot prostheses were able to restore the lost foot length [1-7]. However, recent studies suggest that only some devices are able to restore the effective foot length [8-10]. In one study, the center of pressure $(\mathrm{CoP})$ remained proximal to the end of the residuum in persons with transmetatarsal (TMT) and Lisfranc amputation using toe fillers, insoles, and slipper sockets [9-10]. By contrast, the CoP progressed beyond the end of the residuum in a single person with a TMT amputation using a Blue Rocker Toe-Off Ankle Foot

\footnotetext{
Abbreviations: $\mathrm{AFO}=$ ankle foot orthosis, $\mathrm{BA}=$ below-ankle component, $\mathrm{CoP}=$ center of pressure, $\mathrm{DF} 0=$ dorsiflexion stop at neutral $\left(0^{\circ}\right)$ ankle angle with free plantar flexion, DF10 = stop at $10^{\circ}$ dorsiflexion with free plantar flexion, EVA = ethylene vinyl acetate, FIXED = fixed ankle (locked at neutral), FREE = free ankle motion, $\mathrm{HH}=$ Helen Hayes, $\mathrm{PFA}=$ partial foot amputation, R1 = stiffer forefoot, R2 = compliant forefoot, RE = residual end, TMT $=$ transmetatarsal, VA $=$ Department of Veterans Affairs, vGRF = vertical ground reaction force.

*Address all correspondence to Michael P. Dillon, PhD, BPO(Hons); National Centre for Prosthetics and Orthotics, Musculoskeletal Research Centre, Division of Allied Health, La Trobe University, Bundoora, Victoria 3086, Australia; +61-3-9479-5889; fax: +61-3-9479-5784.

Email: Michael.Dillon@latrobe.edu.au

DOI:10.1682/JRRD.2010.09.0167
} 
Orthosis (AFO) [10] and in persons with Chopart amputation using clamshell prostheses [8-9].

Devices that successfully restore the effective foot length seem to have several common design elements, including a relatively stiff forefoot capable of supporting the amputee's body weight; a large surface area over which pressures caused by loading the prosthetic forefoot can be comfortably distributed to the leg (e.g., an anterior leg shell); and a way of supporting the external moments caused by loading the prosthetic forefoot, which necessitates either rigidly coupling the leg and foot segments of the device so that ankle motion is eliminated (e.g., the clamshell socket) or providing a relatively stiff coupling between the leg and foot segments of the device (e.g., the Blue Rocker Toe-Off AFO) that moderates the degree of dorsiflexion [8-9,11].

In cases where prosthetic intervention restored the effective foot length, persons with PFA were able to progress over and use the prosthetic forefoot, as evidenced by normalization of the ankle plantar flexor moment, restoration of the knee flexor moment following midstance, and normal magnitude and timing of the vertical ground reaction force (vGRF) peaks [12]. Normalization of these parameters has been associated with fewer adaptations aimed at minimizing the socket/foot interface pressure or compensating for atrophy and weakness of the biarticular knee flexors and ankle plantar flexors [12]. Additionally, normalization of the effective foot length is associated with elimination of a "drop off" effect in persons with transtibial amputation for whom the prosthetic forefoot is too short or too compliant [13]. In addition to these observations, an inability to progress over the forefoot has been hypothesized to limit contralateral step length and, in turn, reduce stride length and walking velocity [11,14]. Although the evidence supporting this assertion is limited, these sorts of temporospatial adaptations are widely observed in persons with PFA and seem mechanically sound [15].

While the insights gleaned from these investigations have allowed reasonable hypotheses to be established regarding which features of the partial foot prosthesis are important for restoring the effective foot length, they have not been systematically evaluated. As such, the purpose of this experimental study was to systematically alter forefoot stiffness, ankle joint range of motion, and use of a large leg shell (i.e., an anterior tibial shell) and observe the effect on CoP excursion as well as other parameters known to be affected by changes in CoP excursion, including peak ankle plantar flexor moment, peak knee flexor moment during late stance, timing and magnitude of the second vGRF peak, magnitude of the first vGRF peak on the contralateral side, contralateral step length, and walking velocity. We hypothesized that the effective foot length would be restored with a combination of all three elements: a stiff forefoot, restricted dorsiflexion motion, and an above-ankle anterior leg shell.

\section{METHODS}

\section{Subjects}

Two participants were recruited through prosthetic and orthotic service providers in the Chicago area as well as through several local diabetic foot clinics. Only subjects with an amputation at or proximal to the TMT level were included because a previous investigation did not observe significant reductions in the CoP excursion in persons with metatarsophalangeal amputation [9]. Subjects were excluded from participation if they walked using gait aids, had current ulceration or skin breakdown, or had other neuromuscular or musculoskeletal conditions that affected gait.

\section{Apparatus}

\section{Experimental Prosthesis}

For each participant, an experimental prosthesis was fabricated that allowed randomization of the independent variables: forefoot stiffness, ankle range of motion, and presence of an anterior tibial shell. Design and fabrication of the device used in this study is described in the Appendix (available online only). As shown in Figure 1, the device consisted of a pelite liner, laminated socket, Camber-axis ankle joints (Becker Orthopedic; Troy, Michigan), and a clamshell above-ankle section. The forefoot section included vertical cuts that could be blocked as needed with the use of shims (when all the cuts were open, the foot was more compliant; when the shims were inserted into every second cut, the foot would conform to a radius typical of the nondisabled ankle-foot roll-over shape). The ankle-foot roll-over shape has been defined as the effective geometry that the ankle-foot complex conforms to between initial contact and opposite initial contact [16]. The procedure for making the cuts in the prosthetic foot was similar to that used to determine cut locations in the forefoot of the Shape\&Roll 


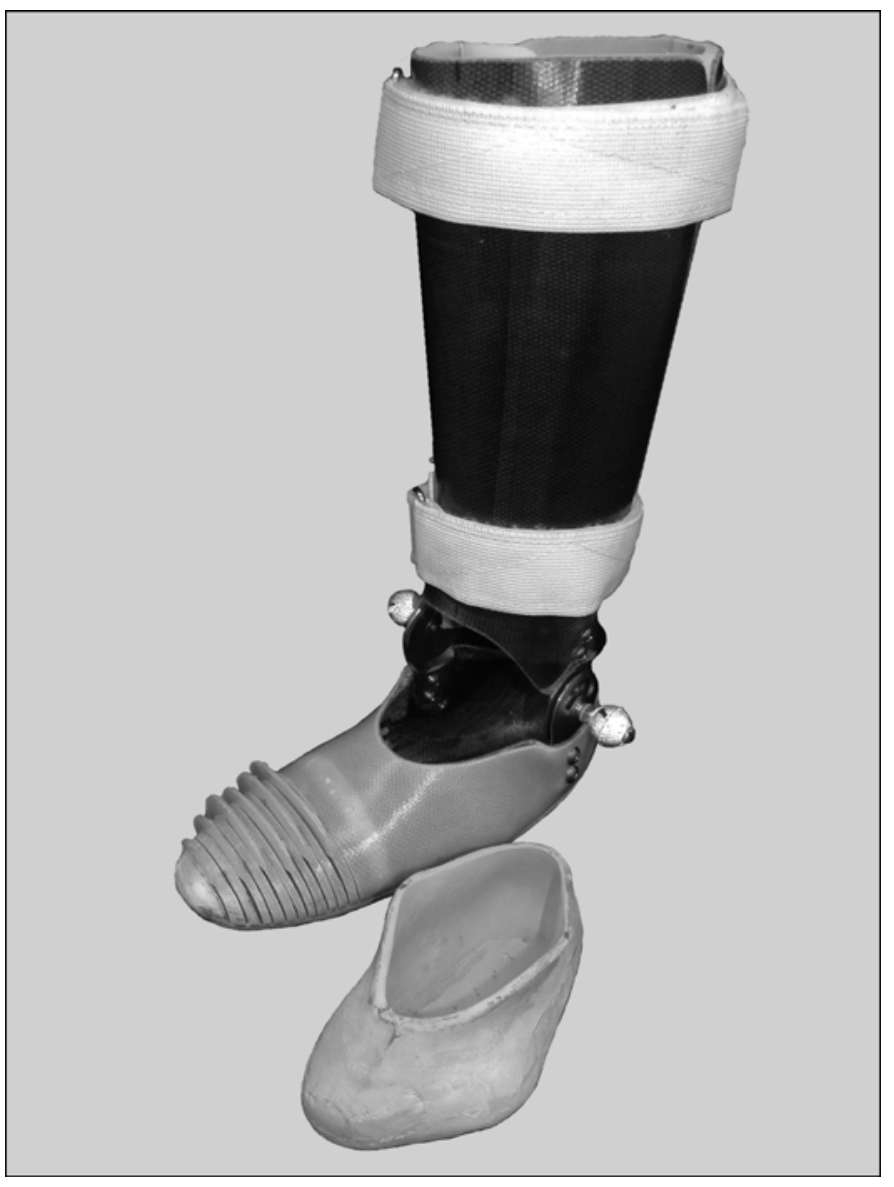

Figure 1.

Experimental prosthesis, including pelite liner, laminated socket, and leg shells with Velcro closures. Camber-axis joints are joined to socket (inferiorly) and anterior leg shell (proximally). Distal forefoot includes series of band saw cuts to allow forefoot to deform to desired roll-over shape. Note the plastic shims that could be fully inserted into every second cut to obtain "stiff" forefoot condition. Removal of all shims created "compliant" forefoot condition. Retroreflective markers were screwed into joint axes during static trials (as pictured). During dynamic trials, medial retroreflective marker was replaced with screw and locking washer.

prosthetic foot [16]. The goal roll-over shape radius was set to 1.25 times the length of the entire foot. This value was used because the nondisabled ankle-foot roll-over shape radius tends to be near 0.19 times the height of the person [17] and a person's foot length is usually about 0.152 times his or her height [18]. The device was designed such that the above- and below-ankle sections could be easily separated and the ankle range of motion and forefoot stiffness could be systematically altered. Measurement and casting for the prosthesis are detailed in the "Procedure" Section.

\section{Gait Laboratory}

An eight-camera, real-time, motion analysis system (Motion Analysis Corporation; Santa Rosa, California) sampled the location of reflective markers at $120 \mathrm{~Hz}$. Synchronized ground reaction force measurements were attained with use of six AMTI force platforms (Advanced Mechanical Technology, Inc; Watertown, Massachusetts) embedded flush in the floor of a $10 \mathrm{~m}$ walkway. The force platforms sampled data at $960 \mathrm{~Hz}$, and as such, these data were resampled to synchronize with the motion data by taking every eighth data point.

\section{Procedure}

This study was approved by the Northwestern University Institutional Review Board, and during the first study visit, written informed consent was obtained from each participant. A qualified prosthetist performed an initial physical assessment that included documentation of cause of amputation, past medical history, and amputation level.

The affected limb of the participant was cast with plaster of paris bandage following indelible marking of bony landmarks and sensitive areas of the residuum and measurement of circumferences and mediolateral dimensions across the ankle and forefoot. Both the leg and residuum were captured in a single cast with a cutting strip placed along the tibial crest and dorsum of the foot to facilitate cast removal. During casting, the foot and leg segments were aligned on a footboard with a profile appropriate for the inside of the footwear worn by the subject during the experiment. The residuum was aligned in a subtalar neutral position, where possible, and the leg segment was vertically oriented. Subjects were cast in a standing position with their typical base of support, as estimated by the prosthetist while observing the participant's gait. Before the cast was removed, vertical orientation lines were marked on the cast in both the coronal and sagittal planes so that alignment of the lower limb could be preserved through the modification and fabrication process. The subject's partial and intact feet were then traced onto a piece of brown paper so that the orientation of the forefoot and toe-out angles could be determined during fabrication of the prosthetic forefoot.

During the second study visit, the prosthesis was fitted, adjusted, and aligned. During static alignment, the ankle joint was both unlocked and locked to ensure that the tibia remained vertical. During dynamic alignment, the sole of the prosthetic foot was adjusted if necessary to 
correct errors in the coronal plane foot-to-floor angle. This adjustment was accomplished by adherence of dense ethylene vinyl acetate (EVA) foam to the sole of the foot to create a wedge.

During the third study visit, subjects presented for a gait analysis at the Department of Veterans Affairs (VA) Chicago Motion Analysis Research Laboratory. Measures of stature and body mass were recorded. Lower-limb joint range of motion [19] and muscle strength [20] testing was conducted according to standard techniques to identify areas of restricted range or muscle weakness that would facilitate interpretation of the gait data.

The independent variables were evaluated in combination to give 10 testing conditions:

1. Below-ankle component with stiffer forefootR1BA.

2. Below-ankle component with compliant forefootR2BA.

3. Above-ankle device with stiffer forefoot and free ankle motion-R1FREE.

4. Above-ankle device with compliant forefoot and free ankle motion-R2FREE.

5. Above-ankle device with stiffer forefoot and dorsiflexion stop at neutral $\left(0^{\circ}\right)$ ankle angle with free plantar flexion-R1DF0.

6. Above-ankle device with compliant forefoot and dorsiflexion stop at neutral $\left(0^{\circ}\right)$ ankle angle with free plantar flexion-R2DF0.

7. Above-ankle device with stiffer forefoot and stop at $10^{\circ}$ dorsiflexion with free plantar flexion-R1DF10.

8. Above-ankle device with compliant forefoot and stop at $10^{\circ}$ dorsiflexion with free plantar flexionR2DF10.

9. Above-ankle device with stiffer forefoot and fixed ankle (locked at neutral)—R1FIXED.

10. Above-ankle device with compliant forefoot and fixed ankle (locked at neutral)-R2FIXED.

We randomized the order of testing of these conditions by using a table of random numbers [21]. Note that a $0^{\circ}$ dorsiflexion stop refers to blocking motion of the ankle so that dorsiflexion cannot be achieved; i.e., ankle motion is stopped when there is a $90^{\circ}$ angle between the foot and shank (sometimes considered a neutral angle of the ankle).

Retroreflective markers were placed on the participant's body, prosthesis, and shoe by the same person on each occasion according to a modified Helen Hayes (HH) marker set [22]. Markers were located on the lateral malleoli (or orthotic equivalent) and lateral femoral condyles, the left and right anterior superior iliac spines, and the sacrum at the superior aspect of the lumbar 5/sacral interface. An additional marker was placed anteriorly on each thigh and shank. The toe and heel markers were positioned on the shoe. The toe marker was positioned on the dorsum of the foot immediately proximal to the end of the residuum and two additional markers were located on the medial and lateral sides of the shoe also proximal to the end of the residuum. The height of the heel marker was adjusted so as to maintain a horizontal relationship with the toe marker. The three markers on the forefoot were used to create a "residual end" (RE) marker set that was used to calculate ankle angles [23]. The RE approach establishes a relationship between the three forefoot markers and the heel marker during a static trial in which the heel of the foot is fully seated within the shoe. This relationship is then used during dynamic trials to establish a virtual heel marker, which is used in the calculation of ankle movement using the standard $\mathrm{HH}$ marker set. The RE marker model has been shown to avoid exaggerated measures of dorsiflexion associated with deformation of the prosthetic forefoot or motion at the pseudojoint between the prosthesis and residuum as well as heel slippage within the shoe [23].

The shank marker was positioned on the anterior shell of the socket in the above-ankle conditions or in the same location directly on the skin for the below-ankle conditions. The shank marker was the only marker that required relocation between certain conditions. The location of markers on the shoe, prosthesis, and leg were recorded by tracing of the base of the marker with an ink pen. The ankle markers were screwed directly into the axis screw of the orthotic ankle joints for all conditions (Figure 1).

Before the commencement of data collection for each condition, a static trial was recorded. During these static trials, additional markers were located on the medial femoral epicondyles, the medial mechanical ankle joint, and the medial malleolus on the intact side. During dynamic trials, medial knee and ankle markers were removed. At the ankle, the medial marker was replaced with a screw and locking washer.

Multiple trials were collected for each of the 10 conditions to ensure that at least three trials with clean force plate strikes were obtained for each limb. A clean force plate strike was defined as a single foot contact with the 
force plate within the force plate boundaries and without contact of the contralateral foot. Subjects were instructed to walk at their normal, comfortable self-selected speed for all trials.

\section{Data Processing}

\section{Calculating Gait Parameters}

EVa RealTime software (Motion Analysis Corporation) was used to determine the three-dimensional position of each marker relative to the laboratory coordinate system during each frame of each trial. The raw coordinate data were filtered with a Butterworth second-order bidirectional low-pass filter with an effective cutoff frequency of $6 \mathrm{~Hz}$, as suggested by Winter [24]. Orthotrak software (Motion Analysis Corporation) was used to calculate kinematics and kinetics of the lower limb joints (except for ankle kinematics), to estimate the timing of gait events, and to output marker and joint center coordinates.

Joint moments were calculated directly by Orthotrak software using an inverse dynamics approach, including the use of anthropometric estimates inherent to the Orthotrak software. Although the inertial properties of a partial foot and a prosthetic intervention clearly create a limb segment with quite different anthropometry, conventional inverse dynamic models for this population have been show to be sufficiently accurate for research questions relating to stance phase [25]. Joint moments were normalized by body mass, yielding units of newtonmeters per kilogram, while vGRF data were normalized by body weight, yielding dimensionless values. vGRF and joint moment data were plotted for the stance phase of walking as a percentage of stance phase.

We calculated the CoP from force plate data by solving the static equilibrium equations of forces and moments applied to the force plate [26]. In early stance phase, we did not calculate the CoP until the vGRF reached a magnitude of $150 \mathrm{~N}$. From earlier experience in this laboratory, we had determined that the CoP was accurate above this vGRF level. The CoP data were then transformed into a shank-based coordinate system, as described in Fatone and Hansen [27]. The forward component of this shank-based coordinate system $\left(\right.$ Shank $\left._{\mathrm{X}}\right)$ is determined from the cross product of a unit vector defining the ankle axis (from the virtual ankle center to the lateral ankle marker) with another unit vector defining the long axis of the shank (from virtual ankle center to virtual knee center) in an order that would result in a vector pointing in the anterior direction. CoP progression along the Shank $\mathrm{X}_{X}$ coordinate was normalized by the shoe length and then plotted as a percentage of stance phase. A horizontal line representing the anterior end of the residual foot was placed on the same plot to help understand whether each condition allowed movement of the CoP beyond the end of the residual foot before opposite initial contact. We created this line by subtracting the residual foot length (from the back of the shoe to the anterior end of the residual foot) from the overall shoe length, dividing the difference by the overall shoe length, and subtracting 0.25 . The 0.25 value is used to remove the scaled portion of the foot from the heel to the ankle, assuming that the ankle is found at about 25 percent of the foot's length with respect to the heel end [18].

\section{Data Analysis}

This experimental study had three independent variables, each with multiple levels: forefoot stiffness (two levels: stiffer, compliant), ankle (restriction) range of motion (four levels: free, locked, dorsiflexion stop at $0^{\circ}$ [neutral] with free plantar flexion, dorsiflexion stop at $10^{\circ}$ with free plantar flexion), and loading area (two levels: above-ankle, below-ankle). The dependent variables were CoP excursion (as a proportion of shoe length), peak ankle plantar flexor moment, peak knee flexor moment following midstance, timing and magnitude of the second vGRF peak, magnitude of the first vGRF peak on the contralateral limb, contralateral step length, stride length, cadence, and walking speed.

Data obtained from multiple trials were averaged for each limb. Given the number of test conditions, reporting only the mean data in the latter figures was necessary.

The "Results" and "Discussion" sections report the consistent patterns of movement that emerged from studying the subjects under different experimental conditions. As such, many of the idiosyncrasies inherent in the gait of individuals have deliberately been overlooked in an attempt to portray a clear understanding of the effects of the different interventions. Where appropriate, movement patterns thought to reflect systematic changes in the experimental conditions have been drawn out.

\section{RESULTS}

We did not observe differences in gait between our two forefoot stiffness conditions (R1 and R2) (Figure 2). 
Post hoc mechanical testing using the BRUCE testing device [28] showed no noticeable change in forefoot stiffness with the cuts open or blocked, suggesting we did not effectively alter forefoot stiffness using this approach. As such, and to simplify presentation of the results, we have only reported results for the "stiff fore-
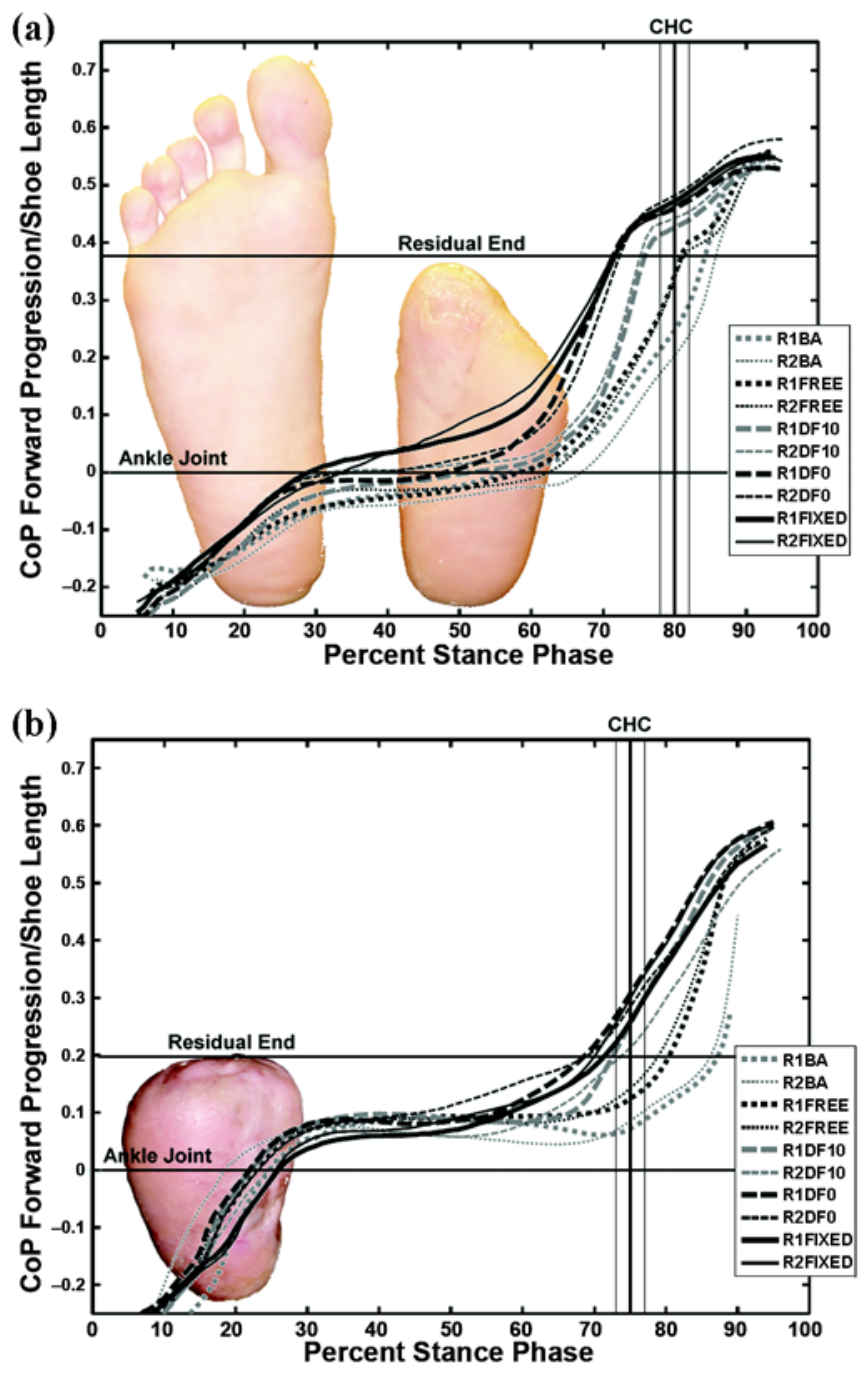

Figure 2.

Center of pressure (CoP) excursion data for (a) subject 1 and (b) subject 2 for both stiff (R1) and compliant (R2) forefoot conditions. CoP was only plotted when magnitude of vertical ground reaction force exceeded $150 \mathrm{~N}$; hence, "gaps" in data at start and end of stance phase. $\mathrm{BA}=$ below-ankle, $\mathrm{CHC}=$ contralateral heel contact $($ mean \pm standard deviation), DF0 $=$ dorsiflexion stop at neutral $\left(0^{\circ}\right)$ ankle angle with free plantar flexion, DF10 = stop at $10^{\circ}$ dorsiflexion with free plantar flexion, FIXED = fixed ankle (locked at neutral), FREE = free ankle motion. foot" condition, which was designed to conform to a radius typical of the nondisabled roll-over shape.

\section{Participant Characteristics}

Two participants were recruited for this investigation. Characteristics have been presented in Table 1 . Physical characteristics of the participants are reported in Table 2. Of note was the reduction in midcalf circumference on the affected limb and reduction in plantar flexion strength (Table 2). Dorsiflexion range was also limited in both participants, particularly with the knee extended (Table 2), indicating that the gastrocnemius muscles were shortened.

\section{Temporospatial Parameters}

Temporospatial data are presented in Table 3. Data on walking speed varied by about 10 percent between the fastest and slowest conditions (Table 3). Within-subject comparison suggests that no systematic differences in walking speed or contralateral step length existed between the test conditions. Subject 2 walked markedly slower than subject 1 as a result of shorter steps with fewer steps per minute. Sound-limb step length was consistently shorter than affected-limb step length across all conditions.

\section{Center of Pressure Excursion}

The CoP excursion data for the affected limbs are presented in Figure 3 (R1: stiff forefoot only). The experimental conditions had similar effects on both subjects in terms of the position of the CoP at contralateral heel contact, the timing of when the $\mathrm{CoP}$ began progressing beyond the ankle, and the rate of that progression. The CoP remained proximal to the distal end of the residuum until after contralateral heel contact in both the BA and FREE conditions. After contralateral heel contact, the CoP progressed beyond the distal end of the residuum so that by the end of stance, a total excursion comparable to that of the other experimental conditions was achieved. When the range of ankle dorsiflexion was constrained (DF0, DF10, FIXED), the CoP was able to progress just beyond the end of the residuum (5\%-10\% of shoe length) by the time contralateral heel contact occurred. No marked differences in the CoP excursion at contralateral heel contract existed between the DF0, DF10, and FIXED ankle conditions.

The different device conditions influenced the timing and rate of CoP excursion anterior to the ankle. In the BA and FREE conditions, substantial progression of the CoP 
Table 1.

Characteristics of participants with partial foot amputation.

\begin{tabular}{cccccc}
\hline Subject & $\begin{array}{c}\text { Amputation } \\
\text { Level }\end{array}$ & $\begin{array}{c}\text { Years Since } \\
\text { Amputation }\end{array}$ & Etiology & Age (yr) & Usual Device \\
\hline 1 & Left transmetatarsal & 23 & Trauma & 26 & $\begin{array}{l}\text { Foot orthosis with toe filler; silicone } \\
\text { slipper socket with toe filler } \\
\text { Foot orthosis with EVA foam toe filler }\end{array}$ \\
\hline EVA = ethylene vinyl acetate, a closed-cell foam. & 3 & $\begin{array}{l}\text { Peripheral } \\
\text { arterial disease }\end{array}$ & 58 & & \\
\hline \hline
\end{tabular}

Table 2.

Measures of anthropometry, lower-limb joint range, and strength for both participants with partial foot amputation.

\begin{tabular}{|c|c|c|c|c|}
\hline \multirow{2}{*}{ Measure } & \multicolumn{2}{|c|}{ Subject 1} & \multicolumn{2}{|c|}{ Subject 2} \\
\hline & Sound Limb & Amputated Limb & Sound Limb & Amputated Limb \\
\hline \multicolumn{5}{|l|}{ Anthropometry } \\
\hline Mass (kg) & 60.7 & - & 75.7 & - \\
\hline Foot Length (m) & 0.23 & 0.142 & 0.276 & 0.138 \\
\hline Shoe Length (m) & 0.262 & - & 0.308 & - \\
\hline Leg Length (m) & 0.80 & 0.79 & 0.88 & 0.88 \\
\hline \multicolumn{5}{|l|}{ Passive Range of Motion $\left({ }^{\circ}\right)$} \\
\hline Thomas Test & 0 & 0 & 10 & 10 \\
\hline Hip Flexion & 135 & 130 & 110 & 120 \\
\hline Hip Abduction (hip extended) & 40 & 40 & 30 & 20 \\
\hline Dorsiflexion (knee 90) & $20 \mathrm{DF}$ & $20 \mathrm{DF}$ & $10 \mathrm{DF}$ & 0 \\
\hline \multicolumn{5}{|l|}{ Muscle Strength ${ }^{*}$} \\
\hline Hip Flexion & 5 & 5 & 5 & 5 \\
\hline Hip Extension (knee $0^{\circ}$ ) & 5 & 5 & 5 & 5 \\
\hline Hip Extension (knee 90) & 5 & 5 & 5 & 5 \\
\hline Hip Abduction & 5 & 5 & 5 & 5 \\
\hline Knee Flexion & 5 & 5 & 5 & 5 \\
\hline Knee Extension & 5 & 5 & 5 & 5 \\
\hline Dorsiflexion (knee 90) & 5 & 5 & 5 & 5 \\
\hline
\end{tabular}

anterior to the ankle was not observed until relatively late in stance phase and the rate of progression was slow compared with the other device conditions, as evidenced by the slope of the CoP excursion curves in Figure 3.
Increased constraint of the ankle through the DF10, DF0, and FIXED conditions caused the CoP to progress anteriorly along the foot length earlier in stance phase and more rapidly than in the BA and FREE conditions. 
Table 3.

Selected temporal and spatial parameters for affected limb of both subjects for stiffer forefoot condition (R1) only.

\begin{tabular}{|c|c|c|c|c|c|}
\hline Parameter & R1BA & R1DF10 & R1DF0 & R1FIXED & R1FREE \\
\hline \multicolumn{6}{|l|}{ Subject 1} \\
\hline Cadence (steps/min) & 112 & 115 & 111 & 113 & 119 \\
\hline \multicolumn{6}{|l|}{ Step Length (m) } \\
\hline Affected & 0.68 & 0.71 & 0.72 & 0.72 & 0.72 \\
\hline Sound & 0.61 & 0.65 & 0.64 & 0.67 & 0.63 \\
\hline Walking Speed (m/s) & 0.91 & 0.90 & 0.92 & 0.85 & 0.92 \\
\hline Cadence (steps/min) & 95 & 93 & 91 & 90 & 95 \\
\hline Stride Length (m) & 1.15 & 1.17 & 1.20 & 1.16 & 1.16 \\
\hline \multicolumn{6}{|l|}{ Step Length (m) } \\
\hline Affected & 0.64 & 0.64 & 0.66 & 0.64 & 0.64 \\
\hline
\end{tabular}

$\mathrm{BA}=$ below-ankle, DF0 $=$ dorsiflexion stop at neutral $\left(0^{\circ}\right)$ ankle angle with free plantar flexion, DF10 $=$ stop at $10^{\circ}$ dorsiflexion with free plantar flexion, FIXED $=$ fixed ankle (locked at neutral), FREE= free ankle motion.

\section{Vertical Ground Reaction Force}

The vGRF data for the affected limbs are presented in Figure 4. For subject 1, the timing of the second vGRF peak occurred earlier (approximately 5\% stance phase) in the BA and FREE conditions than in the FIXED, DF0, and DF10 conditions (Figure 4(a)). In subject 2, the second vGRF peak was markedly smaller than the first vGRF peak (Figure 4(b)).

The vGRF data for the sound limbs are presented in Figure 5. In both subjects, sound-limb loading during early stance was more exaggerated in the BA and FREE conditions than in the DF10, DF0, and FIXED conditions. The timing of the first vGRF peak was delayed in conditions in which ankle motion was restrained (DF10, DF0, FIXED) when compared with the BA and FREE ankle conditions.

\section{Ankle Kinematics}

Ankle kinematics for the affected limbs are presented in Figure 6. In both participants, the degree of dorsiflexion observed during late stance was greater in the BA and FREE ankle conditions than in the constrained conditions (FIXED, DF10, DF0). The FIXED, DF10, and DF0 conditions resulted in comparable kinematic patterns during stance, with only subtle differences in the peak dorsiflexion angle observed. Small delays occurred in the timing of the dorsiflexion peak in the BA and FREE conditions compared with the other conditions.

\section{Ankle Moments}

Ankle moments for the affected limbs are presented in Figure 7. The magnitude of the peak plantar flexor moment progressively increased as the intervention became more extensive (BA to FREE) and dorsiflexion was constrained (DF10, DF0, FIXED). Increases in the magnitude of the plantar flexor moment tended to commence earlier during stance phase as the intervention constrained motion at the ankle. Although evident in both participants, subject 1 portrays the clearest illustration of this change, with the plantar flexor moment occurring earliest in the FIXED condition (45\% stance), followed by the DF0 condition (57\% stance), the DF10 condition (65\% stance), and finally the FREE and BA conditions (63\%-67\% stance) (Figure 7(a)). A similar pattern is observed in subject 2, with clearer distinction between the BA and FREE conditions and less differentiation between the timing of the FIXED and DF0 conditions (Figure 7(b)).

\section{Knee Kinematics}

The pattern of knee motion did not vary between the conditions tested (Figure 8).

\section{Knee Kinetics}

Knee moments for the affected limbs are presented in Figure 9. For both subjects, limiting ankle dorsiflexion (DF10, DF0, FIXED) restored the knee flexor moment (Figure 9(b)) or increased its magnitude (Figure 9(a)) 
(a)
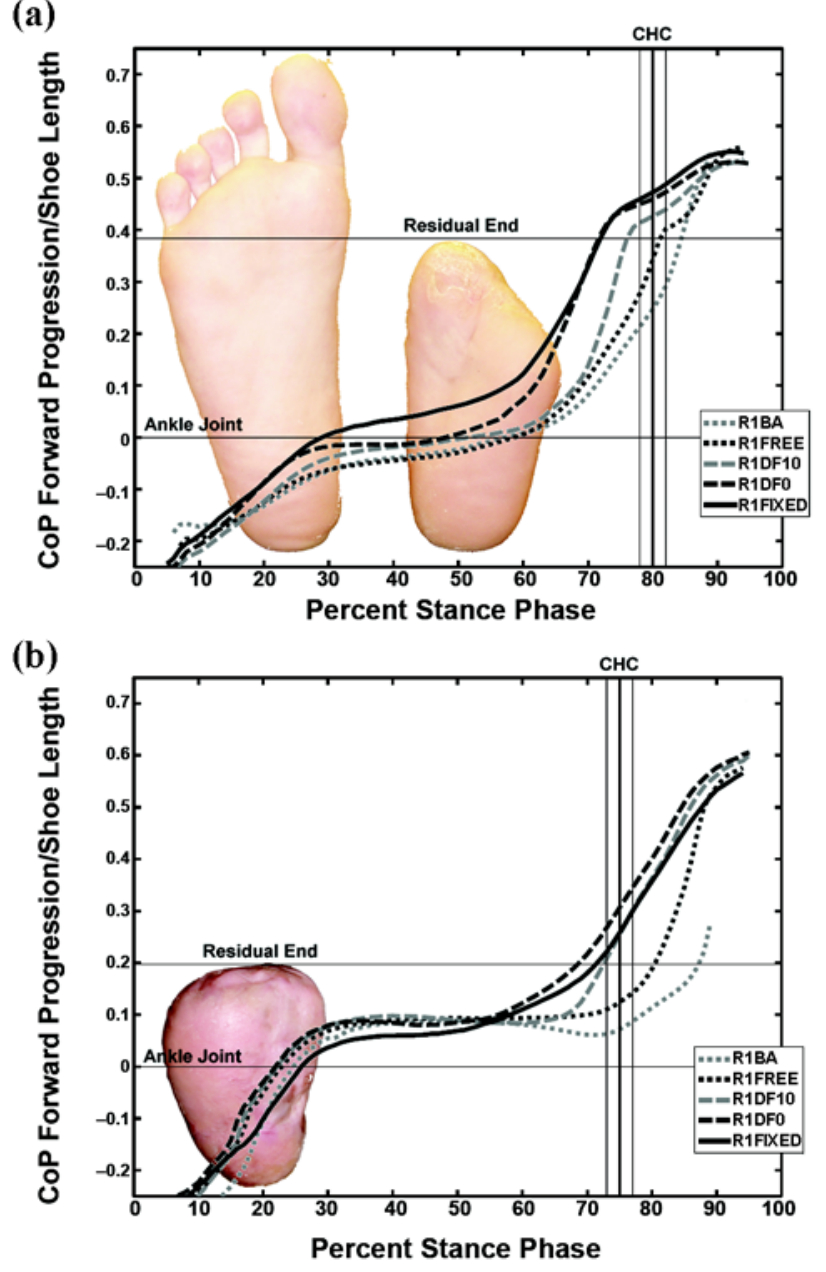

Figure 3.

Center of pressure (CoP) excursion data for (a) subject 1 and (b) subject 2 for stiff forefoot condition (R1) only. CoP was only plotted when magnitude of vertical ground reaction force exceeded $150 \mathrm{~N}$; hence, "gaps" in data at start and end of stance phase. BA = below-ankle, $\mathrm{CHC}=$ contralateral heel contact (mean \pm standard deviation), DF0 = dorsiflexion stop at neutral $\left(0^{\circ}\right)$ ankle angle with free plantar flexion, DF10 = stop at $10^{\circ}$ dorsiflexion with free plantar flexion, FIXED = fixed ankle (locked at neutral), FREE = free ankle motion.

during late stance. As ankle dorsiflexion was increasingly constrained from the DF10 to the DF0 or FIXED conditions, the peak knee flexor moment also increased. This was most dramatic in subject 1 (Figure 9(a)) but clearly evident in both subjects. Constraining ankle dorsiflexion (DF10, DF0, FIXED) delayed the knee moment peak during late stance by about 10-15 percent stance compared with the FREE and BA conditions. The greatest delay was evident in the DF10 condition in both partici-
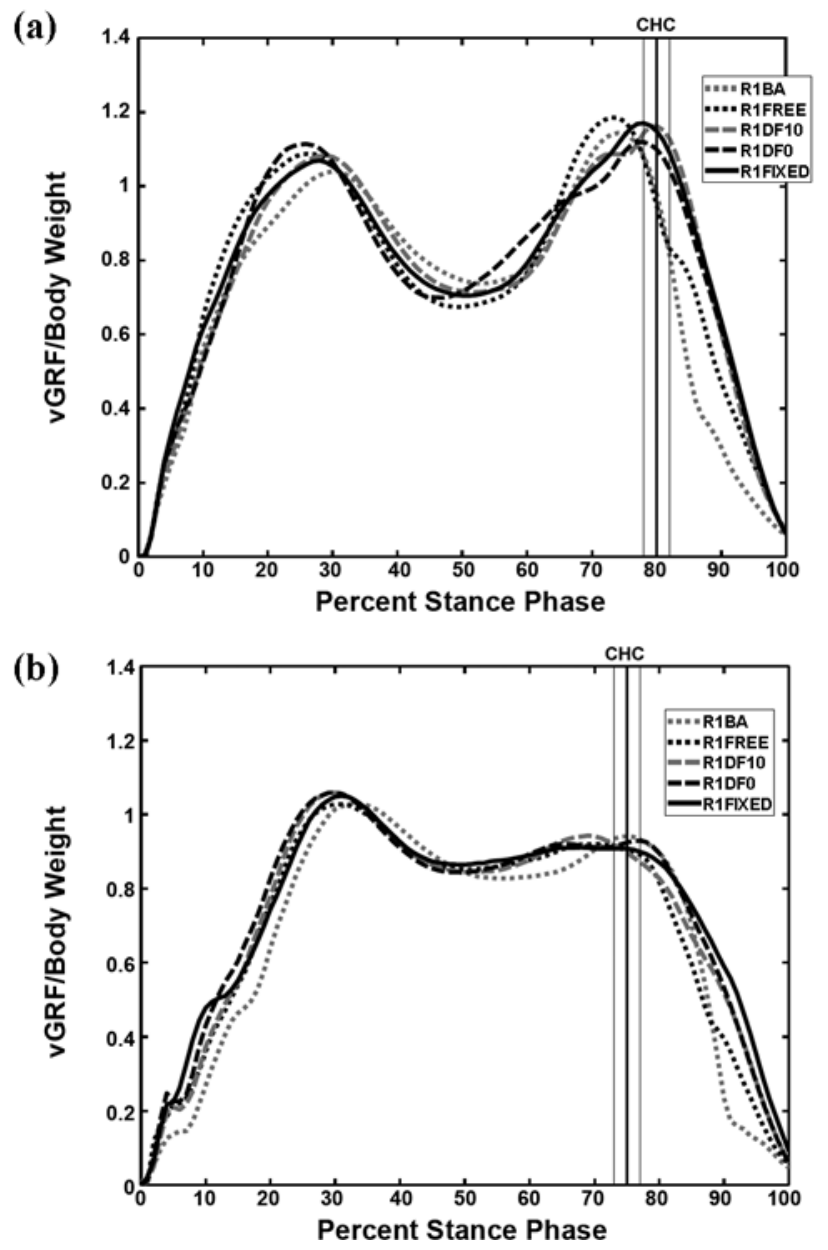

Figure 4.

Vertical ground reaction force (vGRF) data for affected limb of (a) subject 1 and (b) subject 2 for stiff forefoot condition (R1) only. vGRF data has been normalized by body mass. BA = below-ankle, $\mathrm{CHC}=$ contralateral heel contact (mean \pm standard deviation), DF0 = dorsiflexion stop at neutral $\left(0^{\circ}\right)$ ankle angle with free plantar flexion, DF10 $=$ stop at $10^{\circ}$ dorsiflexion with free plantar flexion, FIXED = fixed ankle (locked at neutral), FREE = free ankle motion.

pants, with no difference in the timing of the moment peak between the FIXED and DF0 conditions.

\section{DISCUSSION}

Two quite distinct results were observed, so on this basis, the discussion will be presented in discrete sections examining firstly, the BA and FREE conditions and secondly, the DF10, DF0, and FIXED conditions. These sections will explain the gait patterns observed and a 

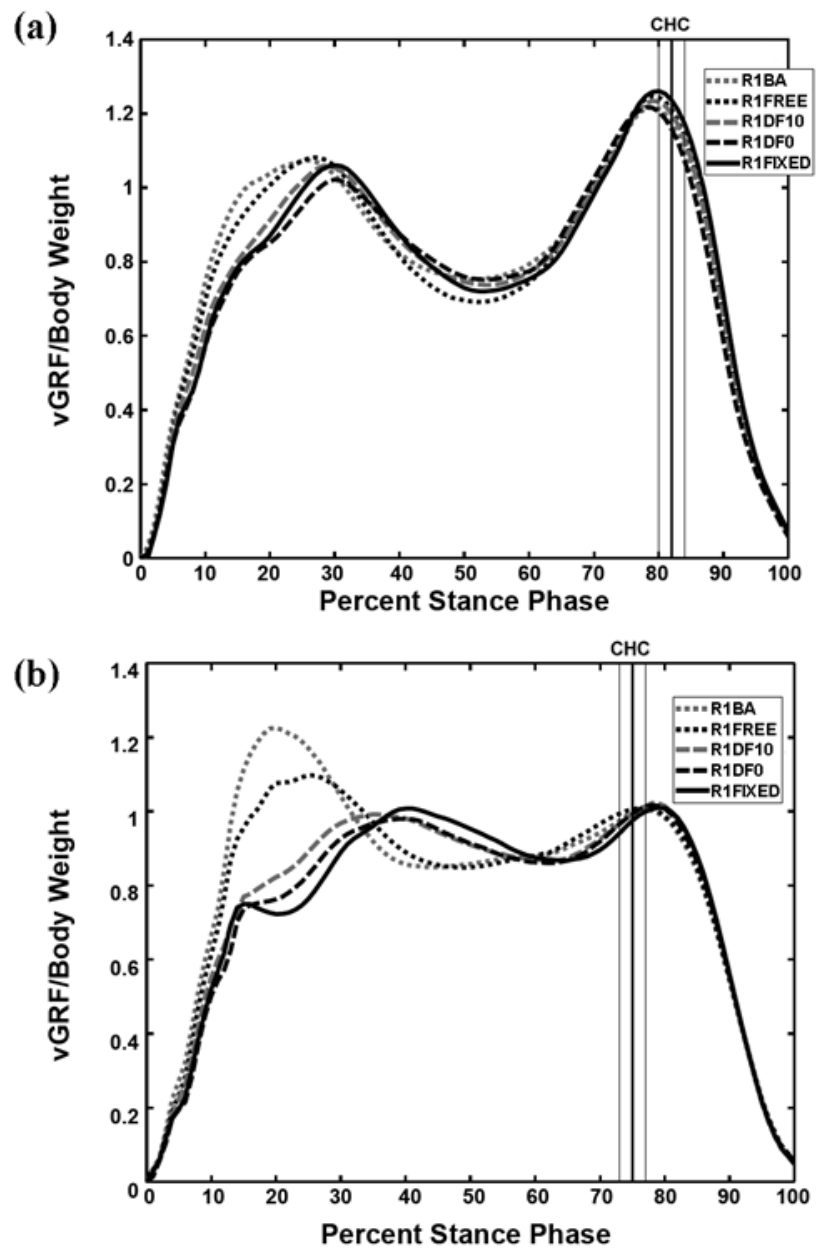

Figure 5.

Vertical ground reaction force (vGRF) data for sound limb of (a) subject 1 and (b) subject 2 for stiff forefoot condition (R1) only. vGRF data has been normalized by body mass. BA = below-ankle, $\mathrm{CHC}=$ contralateral heel contact (mean \pm standard deviation), DF0 = dorsiflexion stop at neutral $\left(0^{\circ}\right)$ ankle angle with free plantar flexion, DF10 $=$ stop at $10^{\circ}$ dorsiflexion with free plantar flexion, FIXED = fixed ankle (locked at neutral), FREE = free ankle motion.

subsequent section will describe how the design of the prosthesis influenced gait. Before launching into the discussion, it is important to establish whether differences in walking speed were evident between the experimental conditions, given that many of the dependent variables are speed dependent. Table 3 indicates no systematic differences in walking speed between the different experimental conditions. Changes in speed varied by about 10 percent between the slowest and fastest conditions. This variability is typical of that observed in persons without limb loss walking on smooth level surfaces at
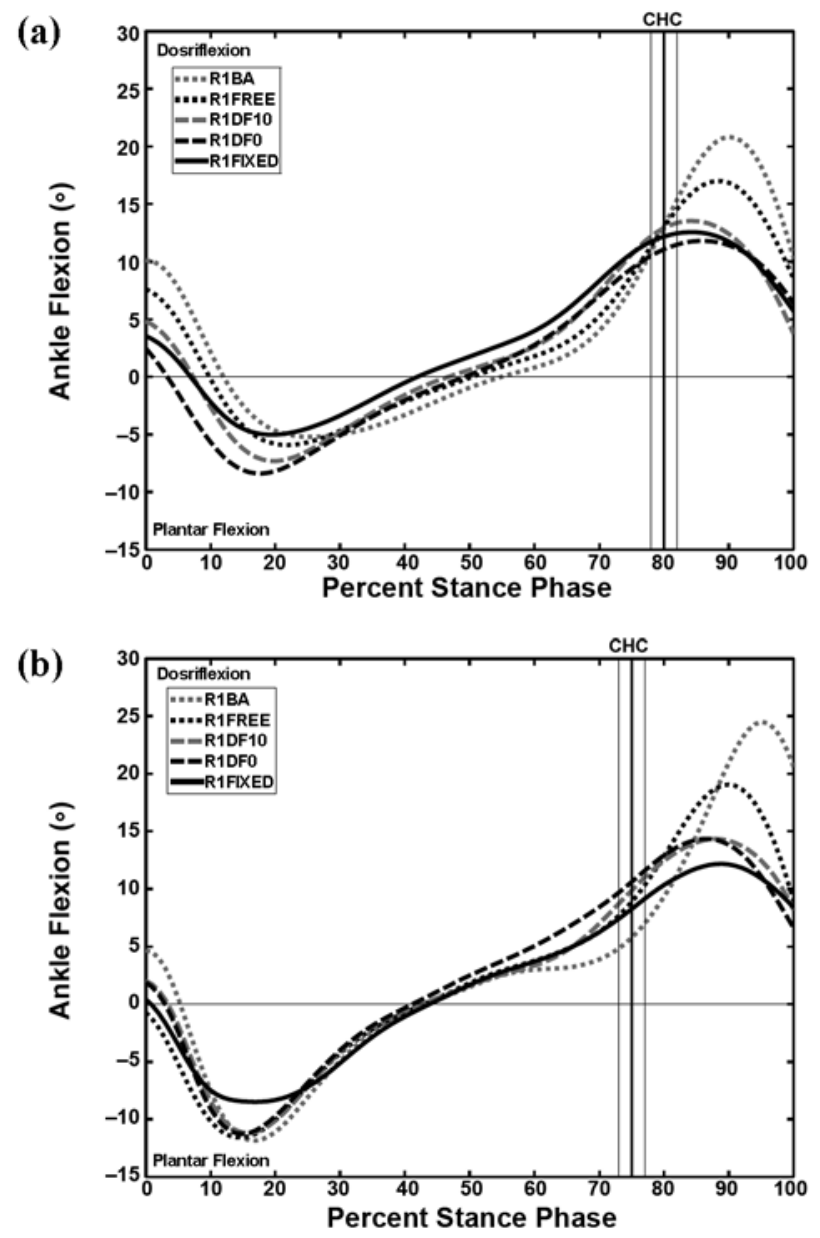

Figure 6.

Sagittal plane ankle kinematic data for affected limb of (a) subject 1 and (b) subject 2 for stiff forefoot condition (R1) only. BA = belowankle, $\mathrm{CHC}=$ contralateral heel contact (mean \pm standard deviation), DF0 $=$ dorsiflexion stop at neutral $\left(0^{\circ}\right)$ ankle angle with free plantar flexion, DF10 = stop at $10^{\circ}$ dorsiflexion with free plantar flexion, FIXED = fixed ankle (locked at neutral), FREE = free ankle motion.

freely chosen walking speeds [29]. Therefore, we can be confident that walking speed was not a covariate affecting analysis of the gait data.

\section{BA and FREE Conditions}

In both the BA and FREE conditions, the CoP remained proximal to the end of the residuum until after contralateral heel contact when the magnitude of the vGRF was rapidly declining (Figures 3 and 4). Interestingly, in subject 1 , whose remnant metatarsals were considerably shorter on the lateral side, the position of the CoP (Figure 3(a)) at the time of the peak vGRF (Figure 4(a)) 

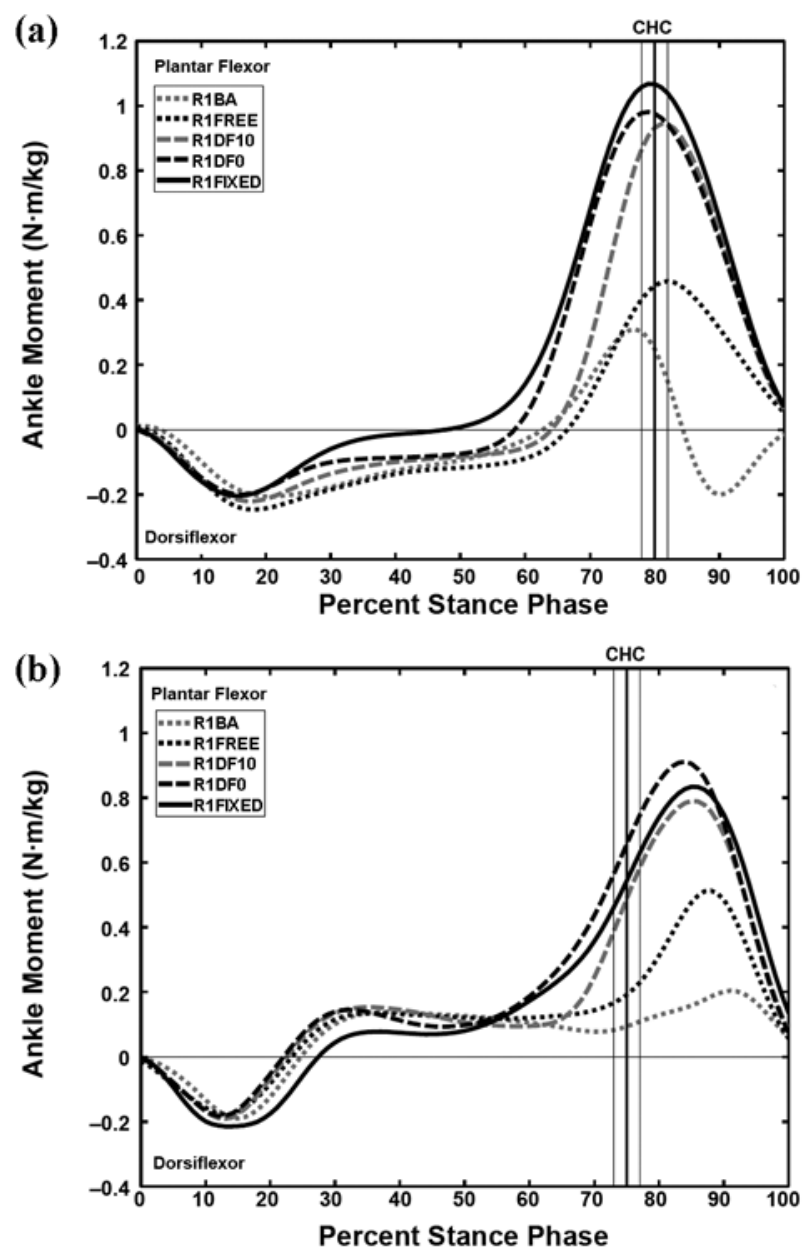

Figure 7.

Internal sagittal plane ankle moments for affected limb of (a) subject 1 and (b) subject 2 for stiff forefoot condition (R1) only. Data has been normalized by body mass and expressed in units of newton-meters per kilogram. BA = below-ankle, $\mathrm{CHC}=$ contralateral heel contact (mean \pm standard deviation), DF0 $=$ dorsiflexion stop at neutral $\left(0^{\circ}\right)$ ankle angle with free plantar flexion, DF10 $=$ stop at $10^{\circ}$ dorsiflexion with free plantar flexion, FIXED = fixed ankle (locked at neutral), FREE = free ankle motion.

remained proximal to the shortest part of the residuum. Maintaining the CoP proximal to the end of the residuum until after contralateral heel contact may be a useful strategy to spare the end of the residuum from the large forces occurring during late stance [12].

As a result of maintaining the $\mathrm{CoP}$ proximal to the end of the residuum, the peak ankle plantar flexor moment was markedly reduced in the BA and FREE conditions (Figure 7), as was the peak knee flexor moment during late stance (Figure 9). Adopting a gait pattern that
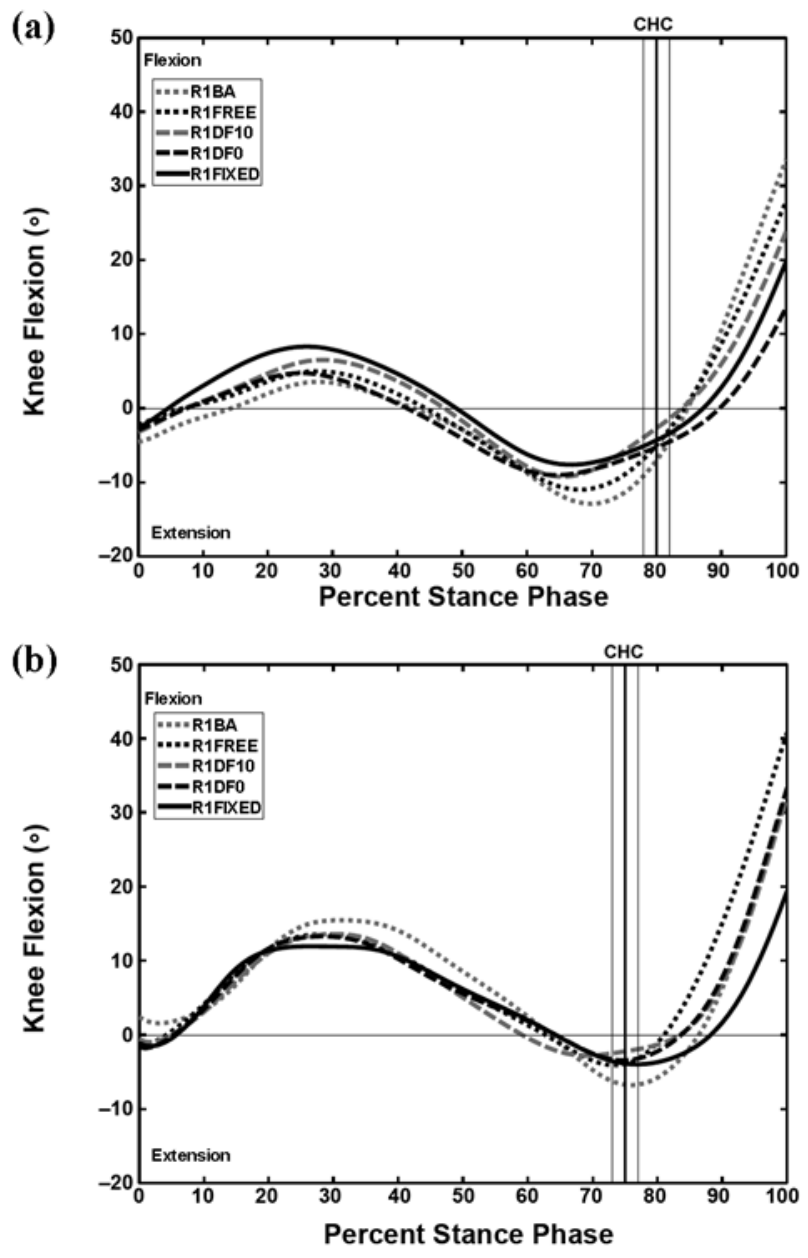

Figure 8.

Sagittal plane knee angles for affected limb of (a) subject 1 and (b) subject 2 for stiff forefoot condition (R1) only. BA = below-ankle, $\mathrm{CHC}=$ contralateral heel contact (mean \pm standard deviation), DF0 = dorsiflexion stop at neutral $\left(0^{\circ}\right)$ ankle angle with free plantar flexion, DF10 = stop at $10^{\circ}$ dorsiflexion with free plantar flexion, FIXED = fixed ankle (locked at neutral), FREE = free ankle motion.

moderates the external ankle and knee moments during late stance may by a useful strategy to compensate for weakness of the triceps surae or to minimize the shear force and pressure on the end of the residuum likely to be caused by concentric contraction of the ankle plantar flexors [12].

The ankle was allowed to progress into increasing degrees of dorsiflexion in the BA and FREE conditions, resulting in an exaggerated dorsiflexion peak as compared with the other conditions (Figure 6). It would appear that restraint of the tibia over the stance foot was limited after midstance. We can be confident in this 

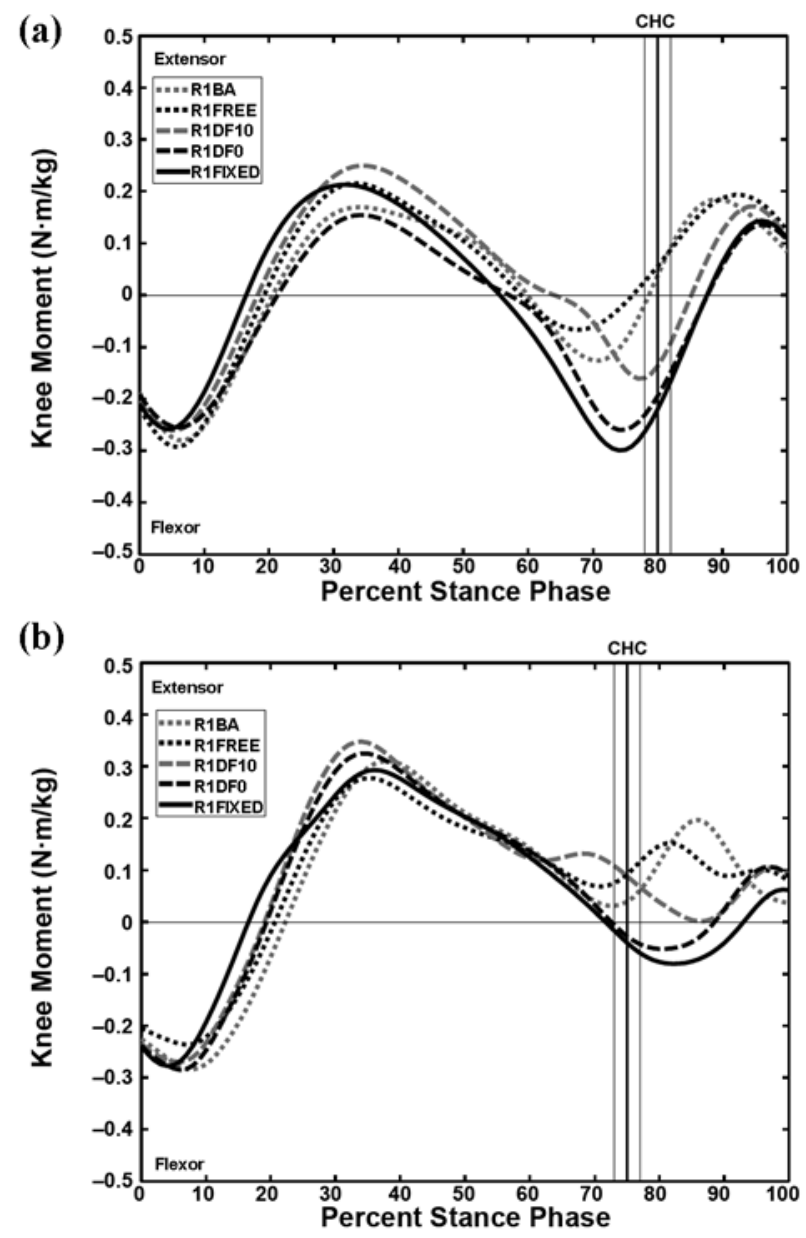

Figure 9.

Internal sagittal plane knee moments for affected limb of (a) subject 1 and (b) subject 2 for stiff forefoot condition (R1) only. Data has been normalized by body mass and expressed in units of newton-meters per kilogram. $\mathrm{BA}=$ below-ankle, $\mathrm{CHC}=$ contralateral heel contact (mean \pm standard deviation), DF0 = dorsiflexion stop at neutral $\left(0^{\circ}\right)$ ankle angle with free plantar flexion, DF10 $=$ stop at $10^{\circ}$ dorsiflexion with free plantar flexion, FIXED = fixed ankle (locked at neutral), FREE = free ankle motion.

assertion, given the small plantar flexor moment observed (Figure 7) and that persons with PFA do not exhibit the large power generation (concentric work) at the ankle [15] that normally restrains tibial progression, limits dorsiflexion, and drives the ankle into plantar flexion. While we did not report ankle power data, we can be assured that concentric work by the ankle plantar flexor musculature was limited given that the internal plantar flexor moment was small (Figure 7). Relatively unrestrained rotation of the tibia over the stance foot was checked by placing the contralateral foot on the ground, exaggerating the first peak of the vGRF on the sound limb compared with conditions in which ankle motion on the affected limb was constrained (Figure 5).

\section{DF10, DF0, and FIXED Conditions}

In the DF10, DF0, and FIXED conditions, the CoP was able to progress beyond the distal end of the residuum before contralateral heel contact (Figure 3). As such, the peak vGRFs were borne by the prosthetic forefoot just distal to the end of the residuum with no differences in the position of the CoP between the different device conditions (Figure 3). This restoration of the effective foot length resulted in larger peak ankle plantar flexor moments (Figure 7) and either restoration of the late stance knee flexor moment (Figure 9(b)) or an increase in its magnitude (Figure 9(a)). Such changes were not observed in the BA and FREE conditions.

In comparison to those in the BA and FREE conditions, the peak dorsiflexion angles were much smaller in the DF10, DF0, and FIXED ankle conditions (Figure 6). However, it was surprising that no differences in peak dorsiflexion angle were observed between the DF10 and DF0 or FIXED conditions (Figure 6), suggesting that the devices did not limit the ankle to the desired joint angle (e.g., DF10, DF0). Although some ankle motion will always be measured by marker-based motion analysis, either due to movement of the leg within the shell or deformation of the prosthetic foot, the total ankle range of $20^{\circ}$ (Figure 6) is double that observed in another investigation of persons wearing nonarticulated clamshell devices [12]. This lends support to the assertion that these articulated devices were unable to limit ankle dorsiflexion as effectively as we might have anticipated.

A number of plausible explanations exist for the exaggerated ankle range and the similarity in the peak dorsiflexion angles between the DF10, DF0, and FIXED conditions. The Camber-axis ankle joints used in the construction of these devices did have some "play" even when we manipulated the device "on-the-bench." With the joint locked in neutral using the appropriate kidneyshaped insert, we were able to measure an average angular change between the foot and leg segment of about $5^{\circ}$ when displacing the leg shell relative to the foot segment. When the joint was dorsiflexed under load, we were also able to create movement between the joint and laminated shell as well as deform the laminated shell around the joint despite reinforcing this area with multiple layers of 
glass fiber during lamination and ensuring the laminate fitted the joint shape intimately. Note that the Camberaxis ankle joints were not designed for this purpose, and as such, we provide these insights to explain aberrations in the data and inform other investigators so they can choose joints designed for the demands of the task.

The different ankle conditions influenced when the $\mathrm{CoP}$ began progressing anteriorly along the length of the foot (Figure 3). In both subjects, the DF10 condition resulted in the CoP progressing anteriorly along the length of the foot later in stance phase than in the DF0 and FIXED conditions (Figure 3), which affected timing of the ankle and knee moments (Figures 7 and 9). The additional dorsiflexion range allowed in the DF10 condition delayed the time when the mechanical ankle joint reached the dorsiflexion stop and only then could the device have an effect on the CoP excursion.

\section{How Prosthesis Design Influenced Gait}

The DF10, DF0, and FIXED conditions were all able to restore the effective foot length because the devices combined the three design elements: a stiff prosthetic forefoot, restriction of ankle dorsiflexion, and an anterior leg shell. In this section, we will look at each of these three design elements in turn and describe why they must be used in tandem to restore the effective foot length and influence other aspects of gait. We will also explain why using just one or two of the design elements does not restore the effective foot length. To ensure these insights are not taken out of context, we wish to make explicit that the following discussion applies only to the gait of persons with TMT and Lisfranc amputations. These insights should not, for example, be extended to the gait of persons with a metatarsophalangeal amputation.

\section{Restriction of Ankle Dorsiflexion}

Results from this investigation make clear that the device must be designed to control the external moments caused by loading the prosthetic forefoot because in persons with TMT and Lisfranc amputations, the calf musculature is not providing this control because of weakness (Table 2), disuse, or discomfort on the distal plantar aspect caused by concentric contraction.

To control the large external moments caused by loading the prosthetic forefoot, the device must restrict ankle dorsiflexion. By virtue of this requirement, the device must extend above the ankle to limit motion between the leg and foot segments. In this investigation, we achieved this by using an anterior tibial shell and a dorsiflexion stop. Other design alternatives may exist that would also work effectively.

Previous investigations have successfully used clamshell prostheses with solid ankle [12], a laminated AFO with dorsiflexion stop [8], or a Blue Rocker Toe-Off AFO [10] to limit dorsiflexion. Each of these differing approaches effectively stiffens the ankle joint, as would the concentric contraction of the ankle plantar flexors in persons without PFA, thereby controlling the external ankle moment. Only when the device can control the external ankle moment can the user load the prosthetic forefoot.

Results from this investigation highlight that the angle at which dorsiflexion is limited influences when the CoP progresses distally along the length of the foot (Figure 3), which in turn influences timing of the ankle plantar flexor (Figure 7) and peak knee flexor moments (Figure 9). It is unclear from this investigation whether subtle differences in the angle at which dorsiflexion is constrained make a meaningful difference to the way persons with PFA walk.

\section{Anterior Tibial Shell}

While the anterior tibial shell was used to incorporate the joints needed to control ankle motion, it also served to comfortably distribute forces caused by loading the prosthetic forefoot.

While other design possibilities may exist, such as a posterior shell AFO with anterior strap, the anterior shell would be the most appropriate design given the system of forces required to control the external dorsiflexion moment and tibial progression over the stance foot. Moreover, the anterior shell provides a much larger surface area over which to distribute forces caused by loading the prosthetic forefoot.

Previous investigations that have successfully restored the effective foot length have all incorporated a large leg shell, in particular, an anterior tibial shell such as that found in clamshell prostheses with solid ankles [12], laminated anterior shell AFOs with dorsiflexion stops [8], and a Blue Rocker Toe-Off AFO [10].

The anterior tibial shell may provide a relatively reliable means to ensure the interface pressures are comfortably distributed to the leg. If the interface pressures are uncomfortable or cause pain, then the user may adopt a gait pattern where large or uncomfortable interface pressure will not be experienced. For example, the user may 
choose to reduce the interface pressures on the anterior leg shell by moderating the external ankle moment. This could be achieved by limiting distal excursion of the CoP until after the peak vGRF occurs or by transferring axial load to the contralateral lower limb before the prosthetic forefoot is loaded.

How large an anterior shell should be to comfortably distribute interface pressures caused by loading the prosthetic forefoot is unknown. One argument is that because the external ankle moments have a large magnitude, a large surface area would be needed to comfortably distribute the interface pressures. A counterargument might be that we already know the interface pressures can be comfortably distributed over a smaller area because it is commonly done in transtibial prosthetic sockets. It is likely that the underlying tissue structures will, in part, determine the shell size and contour. Pressures might be comfortably distributed over a shell high on the tibia, where there is a comparatively large area of soft tissue, as opposed to the same size shell just above the ankle, where the tibial crest and flare are more prominent. Further research assessing pressure distribution with varying device designs may explore this issue.

\section{Forefoot Stiffness}

In this investigation, both forefoot conditions were sufficiently stiff that when combined with an anterior leg shell and restricted ankle motion, the effective foot length was restored (Figure 3). Because we did not effectively alter forefoot stiffness, we cannot draw conclusions about the effect of forefoot stiffness on gait.

One could argue that a prosthesis that incorporates an anterior leg shell and limits dorsiflexion will still not restore the effective foot length when combined with a prosthetic forefoot that is too compliant to support the user's body mass. In this situation, the user would likely not allow the CoP to progress beyond the distal end of the residuum onto the prosthetic forefoot because the prosthetic forefoot would collapse under the load.

How stiff the forefoot should be remains unknown. One hypothesis is that some prosthetic forefeet, such as those made from EVA or a thin carbon-fiber foot plate, would not be stiff enough to support the user's body mass. Devices that have successfully restored the effective foot length have included a forefoot made from a ground down SACH (solid-ankle cushion-heel foot) [12], an extension of the laminated socket out to the "toe break" creating a rigid keel that was then covered in foam
[8,12], or a Blue Rocker Toe Off AFO that was "oversized" so the reinforced section of the foot plate extended as close to the end of the shoe as possible [11].

Results from this investigation suggest that if belowankle devices are being used in persons with TMT or Lisfranc amputation, the forefoot stiffness of these devices need not be a design consideration. In this study, the BA condition had no means to control the external moments caused by loading the prosthetic forefoot because no means to restrain ankle dorsiflexion existed. As such, participants adopted a gait pattern that did not require use of the prosthetic forefoot until after contralateral heel contact when the vGRF was rapidly declining. Given that participants were not able to load the prosthetic forefoot in the BA condition, we would not expect to see changes in their gait pattern as a result of changes in forefoot stiffness. The same understanding can be applied to aboveankle devices that do not restrict ankle dorsiflexion, such as the FREE condition in the present investigation.

\section{Application to Clinical Practice}

This investigation provides insights into the effect of device design on the gait of persons with TMT and Lisfranc amputations. While we recognize that clinicians are often forced to consider competing goals, such as the need to reduce the likelihood of subsequent ulceration and skin breakdown or cosmetic restoration, we hope these insights provide some additional information about the function that can be expected from both below- and above-ankle interventions.

Notwithstanding the limitations of this investigation, we suggest that clinicians prescribe prostheses that incorporate a stiff forefoot, restrict/resist ankle dorsiflexion, and provide a means to comfortably manage the external moments caused by loading the prosthetic forefoot (e.g., an anterior tibial shell) if they wish to restore the effective foot length in persons with TMT and Lisfranc amputations. Incorporating all these design features into the prosthesis is also necessary to control the tibia as it rotates over the stance foot, reduce exaggerated loading of the sound limb during initial contact, and provide a means to control the external moments at the ankle and knee in lieu of the normal power generation by the ankle plantar flexors during late stance.

When fitting below-ankle devices or above-ankle devices that do not restrain ankle motion, there would seem little need to be concerned about the compliance/ stiffness of the forefoot because the CoP remains proximal 
to the end of the residuum until after contralateral heel contact. In these cases, the prosthetic forefoot is not being used to support body weight during single-limb support on the affected limb, and as such, the choice of forefoot material becomes relatively unimportant in terms of the mechanics of gait.

\section{Limitations}

Although we designed a novel device that allowed randomization of the different test conditions, several limitations with the design should be noted.

The prosthesis did not effectively limit ankle range of motion at the desired angle because of play in the joint itself, movement between the joint and the laminated shell, and deformation of the laminated shell around the joint housing. These limitations resulted in measures of dorsiflexion being similar in the DF10, DF0, and FIXED conditions. While this limitation did not hinder our ability to address the original hypothesis that limiting ankle dorsiflexion was necessary to restore the effective foot length, we were unable to determine the angle at which dorsiflexion should be limited or be confident of how much the knee and ankle moments would be affected. This limitation should also be noted when drawing comparison to other investigations using nonarticulated devices (e.g., clamshell prostheses).

We did not effectively create two unique forefoot stiffness conditions, and as such, we are unable to ascertain whether a compliant forefoot would affect the results. As suggested in another study [30], the cuts might have been too close together to function as intended. Future work should investigate a different approach to modulation of forefoot stiffness, such as deepening or widening the cuts.

Participants were given limited time to acclimate to the experimental prosthesis and the individual test conditions. This was a deliberate choice given a number of considerations. We had reservations about providing these unique experimental prostheses to participants for use outside the laboratory where close supervision was not possible. We were mindful of the difficulties previous investigators have had recruiting persons with PFA and had endeavored to create a protocol that was not unduly arduous given the number of test conditions. If each of these test conditions required even a moderate acclimation of a couple of weeks, the experiment would have occurred over many months with visits every couple of weeks. We anticipated that such an approach would not have been feasible for potential participants.

Gauging the extent to which limited acclimation influenced the results is difficult. We can be confident that participants were able to acclimate to some degree given the systematic changes in a number of parameters, as would be expected when manipulating the effective foot length. Certainly our clinical experience suggests that persons are able to adapt fairly quickly to a range of interventions, and this has also been demonstrated in other areas of research [31].

Unfortunately, despite our endeavors to recruit persons with PFA through several diabetic foot clinics and orthotic/prosthetic service providers in the Chicago area, we were only able to recruit two people over a period of nearly 3 years. This certainly limits the external validity and generalizability of our results to a wider population of persons with PFA. We hope that by adopting an approach of reporting consistent and large changes in the gait of these participants, we have avoided reporting idiosyncratic adaptations to our interventions. The limited power of this investigation should be kept in mind when generalizing these results to others with TMT and Lisfranc amputations.

\section{Further Research}

Further work is required on a larger cohort to improve the generalizability of these observations to the wider population of persons with TMT and Lisfranc amputations. This work highlights the need for improvements to the design of the experimental prosthesis to restrict ankle dorsiflexion to the desired angle and create distinct forefoot stiffness conditions. Future investigators may also wish to explore questions about the size of the anterior tibial shell required or the effectiveness of alternative designs. We hope the insights gleaned from this work will contribute to further improvements in prosthetic design.

\section{CONCLUSIONS}

This investigation systematically altered aspects of prosthesis design to understand which design elements were important to restoration of the effective foot length in persons with PFA. Our observations in two persons with PFA suggest that the prosthesis must incorporate each of the following design aspects to restore the effective 
foot length: a stiff forefoot, an anterior leg shell, and restrained dorsiflexion. When such a device was provided to the persons with TMT and Lisfranc amputations, we observed not only restoration of the effective foot length but also less exaggerated dorsiflexion, reduced loading on the sound limb during initial contact, and normalization of the ankle and knee moments during late stance as the device compensated for the limited contribution of the ankle plantar flexors.

\section{ACKNOWLEDGMENTS}

\section{Author Contributions:}

Study concept and design: M. P. Dillon, S. Fatone, A. H. Hansen. Acquisition of data: S. Fatone, A. H. Hansen, M. P. Dillon.

Analysis and interpretation of data: M. P. Dillon.

Drafting of manuscript: M. P. Dillon.

Critical revision of manuscript for important intellectual content:

S. Fatone, A. H. Hansen.

Obtained funding: S. Fatone, A. H. Hansen.

Financial Disclosures: The authors have declared that no competing interests exist.

Funding/Support: This material was based on work supported by the National Institute on Disability and Rehabilitation Research of the U.S. Department of Education (grant H133E030030). The opinions contained in this article are those of the grantee and do not necessarily reflect those of the Department of Education. We acknowledge the use of the VA

Chicago Motion Analysis Research Laboratory of the Jesse Brown VA Medical Center, Chicago, Illinois, and the assistance of Rebecca Stine, MS, with data collection.

Additional Contributions: We thank Steven Gard and Dudley S. Childress for their assistance in obtaining funding and Adam Rozumalski and Michael Schwartz of Gillette Children's Hospital for conducting post hoc forefoot testing of the partial foot devices.

Institutional Review: This study was approved by the Northwestern University Institutional Review Board. During the first study visit, written informed consent was obtained from each participant.

Participant Follow-Up: The authors do not plan to inform participants of the publication of this study. Participants have met with the investigators to discuss the insights from their individual gait analysis.

\section{REFERENCES}

1. Condie DN. Biomechanics of the partial-foot amputations. London (England): Edward Arnold Publishers Ltd; 1970.

2. Mueller MJ, Sinacore DR. Rehabilitation factors following transmetatarsal amputation. Phys Ther. 1994;74(11):1027-33. [PMID: 7972363]

3. Weber D. Partial foot amputation. In: Weber D, Agro M, editors. Clinical aspects of lower extremity prosthetics:
Trans-tibial, symes, and partial foot amputations. Oakville (Canada): Elgin Enterprises; 1991. p. 197-209.

4. Stills ML. Partial foot prostheses/orthoses. Clin Prosth Orthot. 1988;12(1):14-18.

5. Pullen JJ. A low profile paediatric partial foot. Prosthet Orthot Int. 1987;11(3):137-38. [PMID: 3438159]

6. Rubin G. The partial foot amputation. J Am Podiatry Assoc. 1984;74(10):518-22. [PMID: 6491147]

7. Sanders LJ. Transmetatarsal and midfoot amputations. Clin Podiatr Med Surg. 1997;14(4):741-62. [PMID: 9344266]

8. Wening J, Ruzich J, Nunez R. Partial foot device for highlevel ambulators. Acad Today. 2008;4(1):A8-9.

9. Dillon MP, Barker TM. Can partial foot prostheses effectively restore foot length? Prosthet Orthot Int. 2006;30(1): 17-23. [PMID: 16739778] DOI:10.1080/03093640500467480

10. Wilson EJ, Dillon MP. Restoring centre of pressure excursion using a toe off orthosis in a single partial foot amputee. Proceedings of the International Society of Prosthetics and Orthotics, Australian National Member Society; 2005 Nov 2-5; Sydney, Australia. Melbourne (Australia): ISPO ANMS Inc; 2005. p. 31-32.

11. Wilson EJ. Restoring centre of pressure excursion in partial foot amputees using toeoff-style orthoses [dissertation]. [Bundoora (Australia)]: La Trobe University; 2005.

12. Dillon MP, Barker TM. Comparison of gait of persons with partial foot amputation wearing prosthesis to matched control group: Observational study. J Rehabil Res Dev. 2008; 45(9):1317-34. [PMID: 19319756$]$

DOI:10.1682/JRRD.2008.02.0025

13. Hansen AH, Meier MR, Sessoms PH, Childress DS. The effect of prosthetic foot roll-over shape arc length on the gait of trans-tibial prosthesis users. Prosthet Orthot Int. 2006;30(3):286-99. [PMID: 17162519] DOI:10.1080/03093640600816982

14. Ayyappa E. Postsurgical management of partial foot and Syme's amputation. In: Lusardi MM, Nielsen CC, editors. Orthotics and prosthetics in rehabilitation. Boston (MA): Butterworth-Heinemann; 2000. p. 379-93.

15. Dillon MP, Fatone S, Hodge MC. Biomechanics of ambulation after partial foot amputation: A systematic literature review. J Prosthet Orthot. 2007;19(3 Suppl):2-61. DOI:10.1097/JPO.0b013e3180ca8694

16. Sam M, Childress DS, Hansen AH, Meier MR, Lambla S, Grahn EC, Rolock JS. The 'shape\&roll' prosthetic foot: I. Design and development of appropriate technology for lowincome countries. Med Confl Surviv. 2004;20(4):294-306. [PMID: 15688881] DOI:10.1080/1362369042000285937

17. Hansen AH. Roll-over characteristics of human walking with applications for artificial limbs [dissertation]. [Evanston (IL)]: Northwestern University; 2002. 
18. Dreyfuss H. The measure of man; Human factors in design. New York (NY): Whitney Library of Design; 1967.

19. Hoppenfeld S, Hutton R. Physical examination of the spine and extremities. New York (NY): Appleton-Century-Crofts; 1976.

20. Kendall FP, McCreary EK, Kendal HO. Muscles: Testing and function. 3rd ed. Baltimore (MD): Williams and Wilkins; 1983.

21. Moore DS, McCabe GP. Introduction to the practice of statistics. 4th ed. New York (NY): Freeman; 2003.

22. Kadaba MP, Ramakrishnan HK, Wootten ME. Measurement of lower extremity kinematics during level walking. J Orthop Res. 1990;8(3):383-92. [PMID: 2324857] DOI:10.1002/jor.1100080310

23. Dillon M, Hansen AH, Fatone S. Influence of marker models on ankle kinematics in persons with partial foot amputation: An investigation using a mechanical model. J Rehabil Res Dev. 2008;45(4):567-76. [PMID: 18712642] DOI:10.1682/JRRD.2007.08.0129

24. Winter DA. Biomechanics and motor control of human movement. 2nd ed. New York (NY): Wiley; 1990.

25. Dillon MP, Barker TM, Pettet G. Effect of inaccuracies in anthropometric data and linked-segment inverse dynamic modeling on kinetics of gait in persons with partial foot amputation. J Rehabil Res Dev. 2008;45(9):1303-16. [PMID: 19319755] DOI:10.1682/JRRD.2007.11.0190

26. Winter DA. Biomechanics and motor control of human movement. 3rd ed. Hoboken (NJ): John Wiley \& Sons; 2005.

27. Fatone S, Hansen AH. Effect of ankle-foot orthosis on rollover shape in adults with hemiplegia. J Rehabil Res Dev.
2007;44(1):11-20. [PMID: 17551854]

DOI:10.1682/JRRD.2006.08.0090

28. Bregman DJ, Rozumalski A, Koops D, De Groot V, Schwartz M, Harlaar J. A new method for evaluating ankle foot orthosis characteristics: BRUCE. Gait Posture. 2009; 30(2):144-49. [PMID: 19520576] DOI:10.1016/j.gaitpost.2009.05.012

29. Perry J. Gait analysis: Normal and pathological function. Thorofare (NJ): Slack; 1992.

30. Klodd E, Hansen A, Fatone S, Edwards M. Effects of prosthetic foot forefoot flexibility on oxygen cost and subjective preference rankings of unilateral transtibial prosthesis users. J Rehabil Res Dev. 2010;47(6):543-52.

[PMID: 20848367]

DOI:10.1682/JRRD.2010.01.0003

31. Ferris DP, Liang K, Farley CT. Runners adjust leg stiffness for their first step on a new running surface. J Biomech. 1999;32(8):787-94. [PMID: 10433420] DOI:10.1016/S0021-9290(99)00078-0

Submitted for publication September 1, 2010. Accepted October 18, 2010.

This article and any supplementary material should be cited as follows:

Dillon MP, Fatone S, Hansen AH. Effect of prosthetic design on center of pressure excursion in partial foot prostheses. J Rehabil Res Dev. 2011;48(2):161-78.

DOI:10.1682/JRRD.2010.09.0167

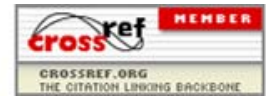


